\title{
Multi-phase mixture modelling of nucleate boiling applied to engine coolant flows
}

\author{
V. Pržulj \& M. Shala \\ Ricardo Software, Ricardo UK Limited \\ Shoreham-by-Sea, West Sussex, UK
}

\begin{abstract}
The paper reports on the use of the homogeneous multi-phase mixture modelling approach to simulate nucleate boiling in low pressure flows. A variant of the RPI (Rensselaer Polytechnic Institute) mechanistic nucleate boiling model provides closures for the wall thermal conditions and mass transfer rates due to phase change in the bulk flow. The bubble departure diameter at the wall and the bubble bulk diameter are identified as the most influential factors and their original model coefficients are modified.

The numerical difficulties due to large density variations associated with phase change are successfully addressed in conjunction with the segregated pressure based SIMPLE (Semi-Implicit Method for Pressure Linked Equations) algorithm.

The nucleate boiling model has been compared against published data from two experiments. The computed and measured values for the wall heat flux and vapour volume fractions are in broad agreement. The model capability is also demonstrated for the engine coolant flow where the conjugate heat transfer problem involving complex engine components is solved.

Keywords: CFD, nucleate boiling, multi-phase mixture, RPI model, engine cooling.
\end{abstract}

\section{Introduction}

Nucleate boiling is the exceedingly effective mode of heat transfer from a heated wall to a liquid. In many liquid cooling systems, ranging from nuclear reactors to electronic devices, a change from single-phase convection to nucleate boiling can effectively provide the desired high rates of heat transfer. The high heat transfer rates (thermal loads) also characterise cylinder blocks and heads of internal 
combustion engines. Their cooling is achieved by pumping a water-glycol mixture through a system of connected coolant passages. Whether nucleate boiling is utilised intentionally or not, the design of efficient liquid cooling systems should avoid departure from the nucleate boiling regime, the boiling crisis. This departure, described by the critical (maximum) wall heat flux (CHF) might lead to the hazardous overheating of wall materials.

Practical CFD modelling of multi-phase flows requires built-in mechanistic models for interfacial mass, momentum and energy transfer between phases. In the past, the majority of published CFD predictions of boiling flows employed the RPI model of Kurul and Podowski [1] for interfacial transfer closures. It was validated against high pressure flows but later some authors [3, 4, 5] adopted this model for low pressure boiling flows. Model improvements have been proposed by [6, 7], while the model extension to conditions close to CHF was reported in [8].

In the majority of CFD simulations $[3-5,9,10]$ the boiling model has been coupled with multi-fluid conservation equations where individual velocity, temperature and other field variables can be solved for each phase. The exception is Bo's work [11], where the homogeneous multi-phase mixture (single fluid) approach was used in conjunction with the author's own mass transfer model.

In the present study, modelling of nucleate boiling based on the RPI model and using the homogeneous multi-phase mixture approach is used. This choice is motivated by uncertainties in modelling interphase transfers and by computational efficiency of the mixture approach. The objective is to explore this overall approach for modelling low pressure coolant flows in internal combustion engines.

\section{Mathematical formulation}

A homogeneous mixture model is adopted in this work. This implies that relative motion between phases is neglected. The modelling equations describing mass, momentum and energy conservation of the mixture have the same form as the single-phase Reynolds Averaged Navier-Stokes equations. A variant of the conventional $k-\epsilon$ model, with imposed bounds on the turbulence time scale is used to model turbulence. The near-wall region turbulence is modelled by enhanced wall functions. In the viscous sub-layer they satisfy corresponding wall-limiting expressions and in the fully turbulent region they are identical to the conventional wall functions. Further details regarding the employed $k-\epsilon$ model and enhanced wall functions can be found in [12].

The transport and thermodynamic properties appearing in the mixture equations depend on the properties of constituent fluid phases $k$ and their volume fractions $\alpha_{k}$. In case of $N_{p h}$ fluid phases, the mixture properties are calculated as:

$$
\phi=\sum_{k=1}^{N_{p h}} \alpha_{k} \phi_{k}=\alpha_{l} \phi_{l}+\alpha_{g} \phi_{g}, \phi=\rho, \mu, \lambda
$$

where $\phi_{k}$ denotes the property value of the constituent phase; in this work of liquid $k=l$ and vapour $k=g$. In the above equation, $\rho, \mu$ and $\lambda$ denote the mixture 
density, dynamic viscosity and thermal conductivity,respectively. The mixture specific heat takes a different form:

$$
C_{p}=\sum_{k=1}^{N_{p h}} X_{k} C_{p, k}=X_{l} C_{p, l}+X_{g} C_{p, g}, X_{k}=\frac{\alpha_{k} \rho_{k}}{\rho}
$$

where $X_{k}$ is the phase mass fraction. Individual phase properties are in general dependent on the temperature. The vapour can be treated as an ideal gas so its density is calculated from the equation of state $\rho_{g}=p /\left(R_{g} T\right)$ where $p$ is the pressure and $R_{g}$ is the gas constant.

The volume fractions are governed by their own transport equations:

$$
\frac{\partial \alpha_{k} \rho_{k}}{\partial t}+\frac{\partial}{\partial x_{j}}\left(\alpha_{k} \rho_{k} U_{j}\right)=\Gamma_{k}, k=l, g
$$

where $t$ is the time, $x_{i}$ are the Cartesian space coordinates and $U_{j}$ are Favreaveraged velocities. The source term, $\Gamma_{k}$, represents the phase mass generation rate due to evaporation and condensation.

The energy conservation equation is defined in terms of total enthalpy, $H=$ $h+0.5 U_{i}^{2}+k$, with $h$ being the specific enthalpy of mixture and $k$ is the turbulent kinetic energy. In order to calculate temperature from the specific enthalpy and vice versa, the specific enthalpy $h$ and its relationship with temperature $T$ needs to be defined. Considering enthalpies of a liquid phase, $h_{l}=C_{p, l} T_{l}$, and a vapour phase, $h_{g}=C_{p, l} T_{s a t}+\Delta h_{g l}+C_{p, g}\left(T_{g}-T_{s a t}\right)\left(T_{s a t}\right.$ denotes the saturation temperature), and setting $T=T_{l}=T_{g}$, the mixture enthalpy can be defined in terms of temperature as:

$$
h=X_{l} h_{l}+X_{g} h_{g}=h_{l, s a t}+X_{g} \Delta h_{g l}+C_{p}\left(T-T_{s a t}\right),
$$

where the specific heat of mixture $C_{p}$ is given by eqn. (2). In the above equation, $\Delta h_{g l}$ signifies the latent heat of evaporation: $\Delta h_{g l}=h_{g, s a t}-h_{l, s a t}$, where $h_{l, s a t}$ and $h_{g, s a t}$, are saturated liquid and vapour enthalpies, respectively. The mixture temperature then becomes

$$
T=T_{\text {sat }}+\frac{h-\left(h_{l, s a t}+X_{g} \Delta h_{g l}\right)}{C_{p}}
$$

\subsection{Mechanistic boiling model}

The mechanistic model presented here provides closures for the wall thermal conditions for the energy and the mass transfer rate $\Gamma_{k}$ in the volume fractions eqn. (3). 
Thermal conditions for the near-wall heat transfer. Considering the wall heat flux $q_{w}$, a general approach is to apportion it to the liquid and vapour phases according to their wall volume fractions $\alpha_{l / g, w}$ :

$$
q_{w}=\alpha_{l, w} q_{w, l}+\alpha_{g, w} q_{w, g}, \alpha_{g, w}=1-\alpha_{l, w}
$$

In the case of sub-cooled nucleate boiling, vapour bubbles, created at the wall nucleation sites and then departed from the wall, are continuously replaced by fresh liquid (quenching) and by new generation of bubbles. Thus the wall liquid fraction is close to one, as the absence of dry wall regions can be assumed. At high wall heat fluxes, the boiling process can move towards CHF conditions. This means that heat transfer between dry wall regions $\left(\alpha_{g, w}>0\right)$ and vapour has to be considered. A phenomenological function $f_{\alpha, l}$ reported in [8]:

$$
\begin{gathered}
f_{\alpha, l}=1-0.5 \exp \left[-20\left(\alpha_{l}-\alpha_{l, c r}\right)\right], \text { for } \alpha_{l} \geq \alpha_{l, c r}, \\
f_{\alpha, l}=0.5\left(\alpha_{l} / \alpha_{l, c r}\right)^{20 \alpha_{l, c r}} \text { for } \alpha_{l}<\alpha_{l, c r}, \alpha_{l, c r}=0.2
\end{gathered}
$$

is used in this work to define the wall liquid fraction $\alpha_{l, w}=f_{\alpha, l}$ in order to model transition from the nucleate boiling to the $\mathrm{CHF}$ regime. In the above expression, $\alpha_{l}$ is a value at the near-wall cell.

Kurul and Podowski's RPI model [1] is used to split the heat flux to the liquid into the single-phase convection $q_{c}$, the wall quenching $q_{q}$ and the evaporation part $q_{e}$ :

$$
q_{w, l}=(1-\Omega) q_{c}+\Omega q_{q}+q_{e}
$$

where $\Omega$ is the fraction of the wall area affected by nucleation sites and quenching, see eqn. (11) below. The wall heat flux expression (6) then reads:

$$
q_{w}=q_{w, d}+\alpha_{l, w}\left[\Omega\left(q_{q}-q_{c}\right)+q_{e}\right], q_{w, d}=\alpha_{l, w} q_{c}+\alpha_{g, w} q_{g, w}
$$

where contribution of convection to the liquid and gas phase is described by the wall diffusion flux $q_{w, d}$. This flux is calculated within the solver using the mixture properties. The liquid convective heat flux $q_{c}$ is calculated in the same way as the wall diffusion flux $q_{w, d}$ except that properties of liquid are used.

The evaporative and quenching flux are given by:

$$
q_{e}=\frac{D_{b w}^{3} \pi}{6} \rho_{g} f_{n} N_{n} \Delta h_{g l}, q_{q}=\frac{2}{\sqrt{\pi}} f_{n} \sqrt{\lambda_{l} \rho_{l} C_{p, l} t_{q}}\left(T_{w}-T_{l}\right)
$$

where $D_{b w}$ is the bubble diameter at departure from the wall, $f_{n}$ and $N_{n}$ are the frequency of nucleation and the number of nucleation sites per unit area, respectively. In the expression for the quenching flux, $t_{q}$ denotes the waiting time elapsed between the departure of a bubble and the nucleation of a new one. It can be estimated as $t_{q}=0.8 / f_{n}$ [1]. The fraction of the wall area affected by nucleation is given as [4]:

$$
\Omega=\min \left(1, \frac{D_{b w}^{2} \pi}{4} N_{n} \eta\right), \eta=4.8 e^{-J a / 80}, J a=\frac{C_{p, l} \rho_{l}\left(T_{s a t}-T_{l}\right)}{\rho_{g} \Delta h_{g l}}
$$

where $J a$ is the Jacob number. 
The closures for the remaining model parameters have been selected based on the assessment of the existing empirical closures reported in literature. For the bubble diameter, the correlation of Tolubinsky, see [5], is modified by introducing an adjustable factor $C_{b w}\left(C_{b w}=0.6\right.$ in the original correlation):

$$
D_{b w}=\min \left(C_{b w} \times 10^{-3} e^{-\Delta T_{s u b} / 45}, 1.4 \times 10^{-3}\right), \Delta T_{\text {sub }}=T_{\text {sat }}-T_{l}
$$

The bubble release frequency and nucleation site density are calculated from Cole's and Lemmert and Chwala's correlations (see for example [5]), respectively:

$$
f_{n}=\sqrt{\frac{4 g\left(\rho_{l}-\rho_{g}\right)}{3 \rho_{l} D_{b w}}}, \quad N_{n}=210\left(T_{w}-T_{\text {sat }}\right)^{1.805}
$$

Interfacial mass transfer. The mass transfer in the bulk flow due to evaporation $\Gamma_{e}$ or condensation $\Gamma_{c}$ is calculated with the help of empirical correlations for the interface heat transfer coefficient around the representative vapour bubble with diameter $D_{b}$ :

$$
\Gamma_{e / c}=\frac{h_{i, e / c} A_{i}\left(T-T_{s a t}\right)}{\Delta h_{g l}}, A_{i}=\frac{6 \alpha_{s g}\left(1-\alpha_{g}\right)}{\left(1-\alpha_{s g}\right) D_{b}}, \alpha_{s g}=\min \left(0.25, \alpha_{g}\right)
$$

where $A_{i}(1 / m)$ is the interfacial area density [1]. For both evaporation and condensation process, the heat transfer coefficient $h_{i}$ is determined from RanzMarshall correlation [3]. The bubble Nusselt number $N u_{b}$ is correlated by Reynolds, $R e_{b}$, and liquid Prandtl, $P r_{l}$, numbers:

$$
h_{i}=\frac{N u_{b} \lambda_{l}}{D_{b}}, N u_{b}=2+0.6 \operatorname{Re}_{b}^{\frac{1}{2}} \operatorname{Pr}_{l}^{\frac{1}{3}}, \operatorname{Re}_{b}=\frac{\rho_{l} U_{b} D_{b}}{\mu_{l}}, \operatorname{Pr}_{l}=\frac{\mu_{l} C_{p, l}}{\lambda_{l}}
$$

The characteristic bubble velocity $U_{b}$ is taken to be the magnitude of the mixture cell velocity. Then, mass transfer rates in eqn. (3) read as $\Gamma_{g}=-\Gamma_{l}=\Gamma_{e}+\Gamma_{c}$

The bubble mean diameter $D_{b}$ has usually been modelled by either a constant, estimated value [8] or as a linear function of local sub-cooling $\Delta T_{s u b}=T_{s a t}-T_{l}$, $[1,3,4]$ with adjustable reference bubble sizes and corresponding sub-cooling temperatures. As shown by [10], these simple approaches are very deficient when compared against the measured bubble size profiles. To accommodate some observed bubble behaviour such as the coalescence, Wintterle et al. [9] adopted models of Basuki et al. and El Jouhary (see [9]):

$$
D_{b}=\left(\frac{6 \alpha_{g}}{\pi N\left(\alpha_{g}\right)}\right)^{\frac{1}{3}}, N=\left[1+\exp \left(\frac{\alpha_{g}-0.015}{0.004}\right)\right]^{-1}\left(N_{0}-N_{1}\right)+N_{1}
$$

where $N_{0}=1.1 \cdot 10^{7}$ and $N_{1}=3.0 \cdot 10^{5}$ are the number densities $\left(1 / \mathrm{m}^{3}\right)$ corresponding to the very low and very high vapour fractions $\alpha_{g}$. 


\section{Numerical framework}

The boiling model has been implemented into an advanced, commercial CFD solver, VECTIS-MAX. The governing equations are discretised over general unstructured (polyhedral) grids employing an advanced collocated Finite Volume Method, [12].

The pressure based, segregated SIMPLE-like solution algorithm [2] ensures efficient pressure-velocity-density coupling. Considering the compressible gas flow, in addition to the pressure and velocity corrections, the density corrections are introduced and defined in terms of the pressure corrections as $\rho^{\prime} \propto C_{\rho} p^{\prime}$, where $C_{\rho}$ is the density derivative over pressure. In the case of a multi-phase mixture, $C_{\rho}$ can be calculated from the mixture sonic speed $c_{m}$ :

$$
C_{\rho}=\frac{1}{c_{m}^{2}}=\rho\left(\frac{\alpha_{g}}{\kappa R_{g} T \rho_{g}}+\frac{\alpha_{l}}{\rho_{l} c_{l}^{2}}\right) \approx \frac{\rho}{\rho_{g}} \frac{\alpha_{g}}{R_{g} T} .
$$

where $\kappa=1.4$ is the isentropic exponent. The above simple formulation for the mixture density derivative over pressure has been effective in handling large density variations associated with phase change.

\section{Results and discussion}

The nucleate boiling model is first validated against published data from two experiments. To assess the model capability for automotive applications, real engine coolant flow simulations are then presented.

The initial validation results identified the bubble departure diameter $D_{b w}$ and the bubble bulk diameter $D_{b}$ as the most influential model parameters. In order to reproduce the experimental trends, it was necessary to adjust their correlations. Therefore, the adjustable factor in eqn. (12) was set to $C_{b w}=1.2$, while the number densities in the expression for $D_{b}$, eqn. (16), were tuned to the values $N_{0}=1.6 \cdot 10^{7}$ and $N_{1}=3.0 \cdot 10^{6}$.

Horizontal channel flow. Robinson [13] tried to replicate engine flow conditions in a cylinder head cooling passages by using a rectangular channel $(241 \mathrm{~mm}$ long, $16 \mathrm{~mm}$ wide and $10 \mathrm{~mm}$ high) with a heated section $\left(10 \times 50 \mathrm{~mm}^{2}\right.$, positioned $76 \mathrm{~mm}$ downstream from the flow inlet) at the bottom wall, see fig. 1 . The

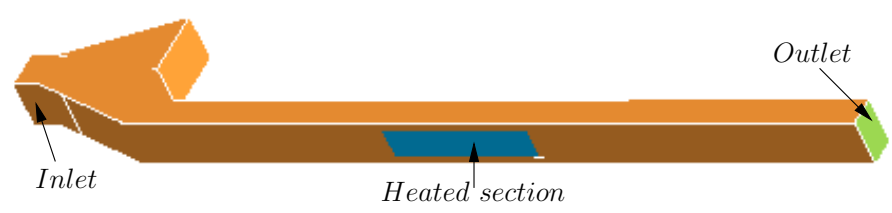

Figure 1: Simulated channel geometry and location of the heated section. 
heated surface (aluminium alloy) was machine finished and can be considered as smooth. The working fluid was a mixture of $50 \%$ water and $50 \%$ ethylene glycol by volume.

The present results were obtained for the sets of test data with the inlet liquid temperature $T_{i n l}=363 \mathrm{~K}$ and with two mass flow rates corresponding to the inlet velocities of $0.25 \mathrm{~m} / \mathrm{s}$ and $1.0 \mathrm{~m} / \mathrm{s}$. Within these sets the pressure values were 1,2 and 3 bars (the corresponding saturation temperatures are $381 \mathrm{~K}, 401 \mathrm{~K}$ and $415 K$, respectively). These pressure values were specified as the outlet boundary conditions. On the heated section, a constant temperature (known from the experiments) was specified. Other walls were considered adiabatic.

The calculated wall heat flux values can be compared against the measured ones as shown in fig. 2. Very good agreement has been obtained for the inlet velocity $0.25 \mathrm{~m} / \mathrm{s}$, fig. 2 (a). The heat flux values for the high velocity of $1 \mathrm{~m} / \mathrm{s}$ are significantly under-predicted as shown in fig. 2 (b). This model behaviour can be partly explained by evident under-prediction of the single-phase heat fluxes before the onset of nucleate boiling. Thus the effect of turbulence modelling and wall functions should be anticipated. Another factor is the dependence of the bubble departure size on the local velocity [3] which is not taken into account by eqn. (12).

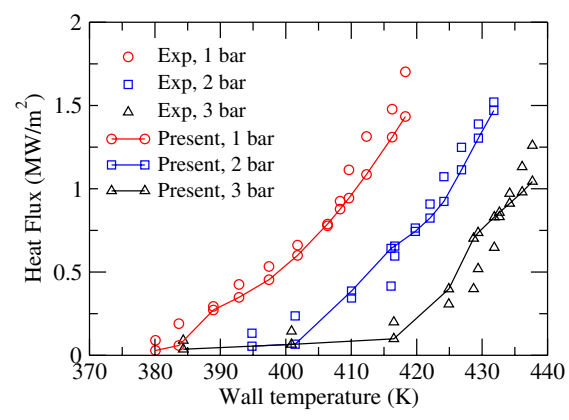

(a) Inlet velocity: $0.25 \mathrm{~m} / \mathrm{s}$

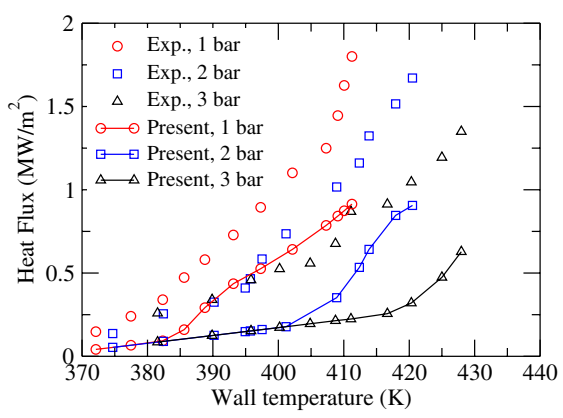

(b) Inlet velocity: $1.0 \mathrm{~m} / \mathrm{s}$

Figure 2: Channel flow, inlet temperature $363 \mathrm{~K}$. Comparison of the predicted wall heat fluxes against measured data from [13].

An example of the computed flow variable fields is depicted in fig. 3. The selected case has the high wall temperature and heat flux which resulted in the large amount of vapour near the heated wall. Consequently, the velocity profiles are distorted when compared to the profiles upstream of the heated section.

Upward flow in a vertical annulus. Bae et al. [5] benchmarked their full EulerEuler (two-fluid) multi-phase model with an interfacial area equation using the experiment from the Seoul National University. The sub-cooled boiling of water 


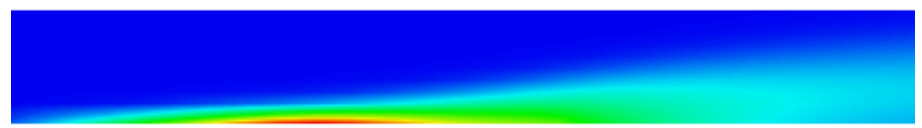

(a) Temperature, from $363 \mathrm{~K}$ (blue) to $414 \mathrm{~K}$ (red)

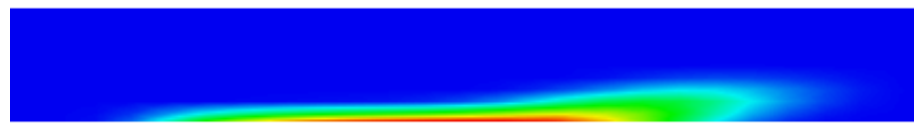

(b) Vapour volume fraction, from 0 (blue) to 0.75 (red)

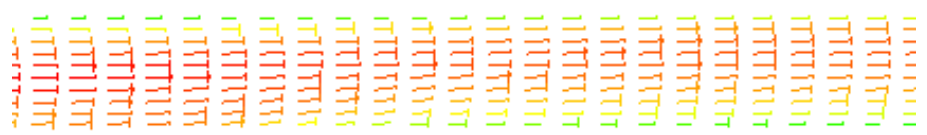

(c) Velocity vectors, from $0.009 \mathrm{~m} / \mathrm{s}$ to $0.33 \mathrm{~m} / \mathrm{s}$

Figure 3: Channel flow, inlet temperature $363 \mathrm{~K}$, velocity $0.25 \mathrm{~m} / \mathrm{s}$. Computed variable fields in the symmetry plane for $p=1 \mathrm{bar}, T_{w}=418 \mathrm{~K}$.

in a vertical upward flow through a concentric annulus was investigated under constant wall heat flux conditions along the heated inner tube section. The total length of the annulus was $3.06 \mathrm{~m}$. The inner tube consisted of the inflow unheated, central heated and outflow unheated section, each $0.5,1.87$ and $0.69 \mathrm{~m}$ long, respectively. The inner and outer annulus radii were $R_{\text {in }}=9.5$ and $R_{\text {out }}=20 \mathrm{~mm}$. The radial distribution of the vapour fraction was measured at three axial positions along the heated section.

For the present validation, a test case with the following conditions has been selected: the mass flow rate $342.207 \mathrm{~kg} /\left(\mathrm{m}^{2} \mathrm{~s}\right)$ (applied as the outlet boundary condition), the wall heat flux at the heated section $q_{w}=212.706 \mathrm{~kW} / \mathrm{m}^{2}$, the pressure 1.21 bar (applied as the inlet boundary condition) and the inlet sub-cooling $T_{\text {sat }}-T_{\text {inl }}=21.695 \mathrm{~K}$. Steady-state simulations are carried out for quarter of the annulus. Apart from the heated inner section, all other walls are assumed adiabatic. As the heat flux is prescribed at the heated section, the wall temperature is calculated with an iterative procedure which ensures that the heat flux eqn. (9), expressed as $q_{w}=F\left(T_{w}\right)$, is satisfied.

The predictions of the radial profile of the vapour fraction at the exit of the heated section are depicted in fig. 4(a). The axial profile of the average vapour fraction is shown in fig. 4(b). The present profiles can be compared with the measured data as well as with two-fluid model predictions from [5]. Two present profiles are presented: the first with the original densities numbers in eqn. (16) for the bubble bulk diameter and the second with modified values of these numbers. The modified densities numbers have improved predictions of the average axial profile with reference to the measured data. Considering the local radial predictions, the present approach over-predicts the amount of vapour near the wall and under-predicts it in the bulk flow. Calculated temperature and vapour fraction fields as obtained with adjusted density numbers are plotted in fig. 5 for the whole solution domain. 


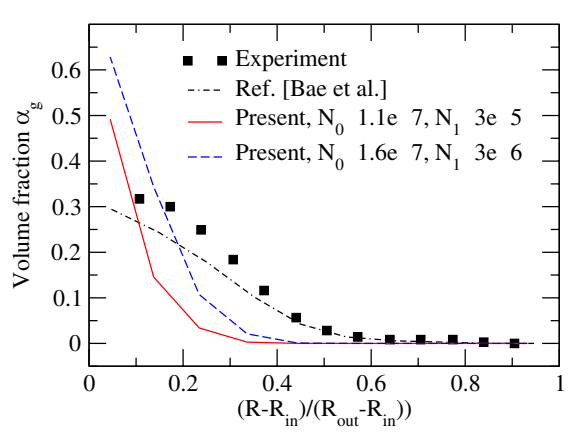

(a) Local radial profile.

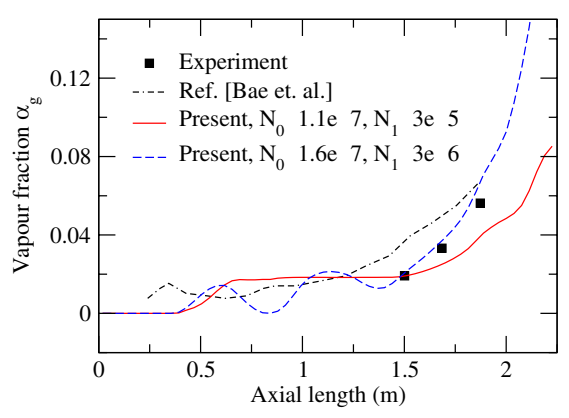

(b) Average axial profile.

Figure 4: Annular flow. Computed and measured vapour volume fraction profiles. (Zero value on the $x$-axes in (b) denotes beginning of the heated section.)

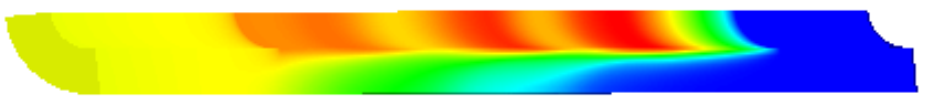

(a) Temperature, from $356 \mathrm{~K}$ (blue) to $376.3 \mathrm{~K}$ (red).

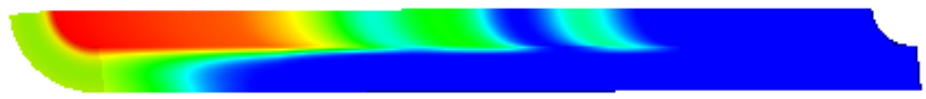

(b) Vapour fraction, from 0 (blue) to 0.69 (red).

Figure 5: Vertical annulus (flow direction from the right to the left). Predicted distribution of temperature and vapour fraction.

In the first half of the heated section the wall temperature experiences an oscillatory pattern which corresponds to the vapour fraction pattern.

Engine coolant flow. The CFD solution domain needs to include the complete engine assembly consisting of typical components such as the cylinder head, head gasket, engine block, cylinder liners and coolant jacket. These components (except liners) are shown in fig. 6 for the selected engine type. The coolant jacket represents the fluid domain while other components define separate solid material domains, each with their own thermal properties.

To make the numerical approach practical, automatic meshing of the solution domain is required because of the very complex geometry. For the present simulation, the Cartesian cut-cell grid, having around 3.3 million cells, has been generated by VECTIS-MAX mesher. This mesher delivers fast meshing without resorting to the boundary surface grid generation.

As part of the steady Conjugate Heat Transfer (CHT) simulation, the mass, momentum, turbulence and volume fraction equations are solved for the fluid domain (coolant flow). The energy equation is solved over a global solution domain 


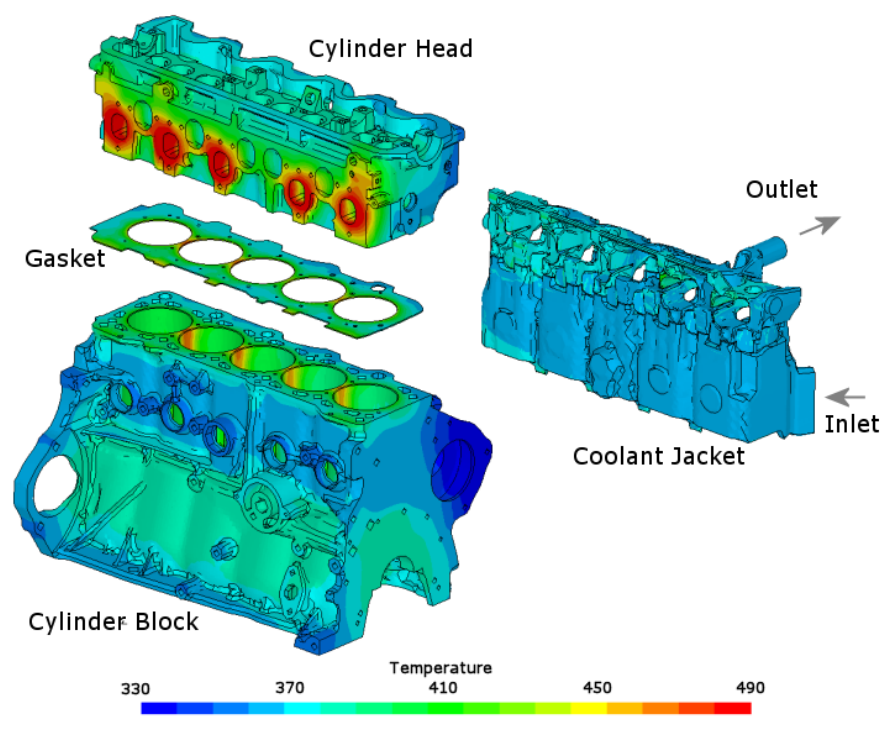

Figure 6: Engine cooling. Temperature distribution at surfaces of fluid (coolant jacket) and solid (cylinder head, gasket and cylinder block) materials participating in the CHT simulation with boiling model.

(containing all participating fluid and solid domains) in a fully implicit and conservative manner. For this, conformal numerical grids at fluid-solid interfaces are provided. At the coolant inlet the mass flow rate $(2.8 \mathrm{~kg} / \mathrm{s})$ and temperature $(363 \mathrm{~K})$ are specified; at the outlet fixed static pressure (1.01bar) is maintained. The external heat transfer coefficient and temperature describe heat transfer at the solid surfaces exposed to the environment. At the gas side cylinder surfaces the heat flux distribution is prescribed.

Fig. 6 presents temperature field at the surfaces of each material domain as calculated with the boiling model. These surfaces are either fluid/solid or solid/solid interfaces or external boundaries.

The effect of the boiling model on the temperature levels is clearly demonstrated in fig. 7. Here, one can compare temperature on the cylinder head as predicted with and without the nucleate boiling model. Inclusion of the boiling model reduces temperature levels. In this case, the maximum temperature without the boiling model is $576.8 \mathrm{~K}$, while the maximum temperature with the boiling model reaches $550.5 \mathrm{~K}$. The maximum temperature of coolant is also reduced from 466.6 to $425.2 \mathrm{~K}$. As expected, the temperature reduction indicates presence of a certain amount of vapour. This amount can be quite large as shown in fig. 8. The figure depicts the coolant passage region in which the near-wall vapour fraction has the high value, around 0.3 . The spot with the maximum value of 0.61 is not shown in the above figure. Identification of such local spots where departure from 

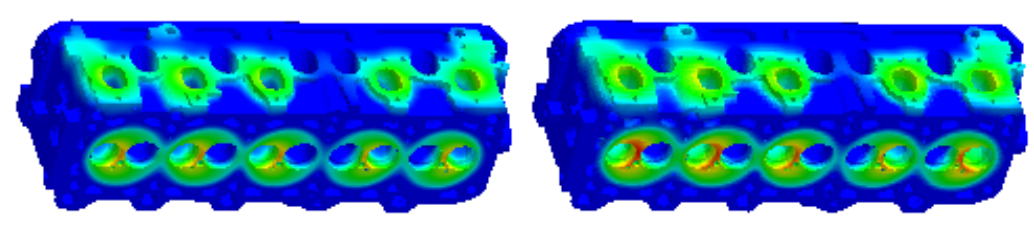

Figure 7: Engine cooling. Comparison of the surface temperature on the cylinder head as computed with (left) and without (right) boiling model, values from $352 \mathrm{~K}$ (blue) to $576.8 \mathrm{~K}$ (red).

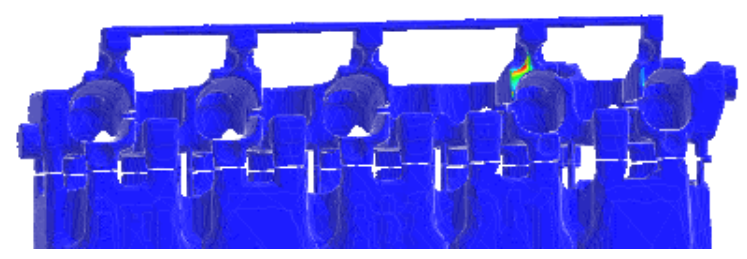

Figure 8: Engine cooling. Near-wall vapour fraction distribution in the coolant passage region with the large amount of vapour, range: from 0 (blue) to 0.3 (red).

nucleate boiling might lead to overheating of the wall materials is useful for design of engine cooling systems.

\section{Conclusion}

The capability of the multi-phase mixture modelling approach, supplemented with the mechanistic nucleate boiling model, has been assessed for CFD predictions of low pressure coolant flows in internal combustion engines. The nucleate boiling model is based on the popular RPI model. Among a number of modelling parameters, the bubble departure diameter at the wall and the bubble bulk diameter are the most influential and required adjustments. In comparison to the original RPI model, the wall heat flux partitioning is modified to account for the possible heat transfer from the wall to the gas phase.

Two boiling flow experiments are used to benchmark the model. The computed and measured values for the wall heat flux and vapour volume fraction are found to be in broad agreement. The CHT simulation of an engine cooling system has shown reasonable model behaviour. Bearing in mind uncertainties in the modelling of nucleate boiling, the present approach can be seen as a good compromise in terms of the accuracy and computational efficiency. 


\section{References}

[1] Kurul, N. \& Podowski, M., Multidimensional effects in forced convection subcooled boiling. Proceedings of the 9th International Heat Transfer Conference, Jerusalem, Israel, 1-BO-04, pp. 21-26, 1990.

[2] Ferziger, J. \& Peric, M., Computational Methods for Fluid Dynamics. Springer: Berlin, 1997.

[3] Koncar, B. \& Mavko, B., CFD simulation of subcooled flow boiling at low pressure. Intl. Conf. Nuclear Energy in Central Europe 2001, Portoroz, Slovenia, pp. 208.1-208.8, 2001.

[4] S. Narumanchi, D.B., A. Troshko \& Hassani, V., Numerical simulations of nucleate boiling in impinging jets: Application in power electronics cooling. Int J Heat and Mass Transfer, 51, pp. 1-12, 2008.

[5] B-U. Bae, H.Y.Y., Euh, D.J., Song, C.H. \& Park, G.C., Computational analysis of a subcooled boiling flow with a one-group interfacial area transport equation. J Nuclear Science and Technology, 45(4), pp. 341-351, 2008.

[6] N Basu, G.W. \& Dhir, V.K., Wall heat flux partitioning during subcooled flow boiling: Part 1 - Model development. ASME J Heat Transfer, 127, pp. 131140, 2005.

[7] G. Yeoh, M.H. \& Tu, Y., Improved wall heat partition for subcooled boiling flows. 6th Int. Conf. Multiphase Flow ICMF 2007, Leipzig, Germany, 2007.

[8] Koncar, B. \& Mavko, B., Simulation of boiling flow experiments close to CHF with the Neptune-CFD code. Science and Technology of Nuclear Installations, 2008, p. 8, 2008.

[9] T. Wintterle, Y.E., E. Laurien \& Menter, F., Numerical simulation of a subcooled boiling flow in a nuclear reactor bundle geometry. 11th Workshop on Two-Phase Flow Predictions, Merseburg, Germany, 2005.

[10] Yeoh, G. \& Tu, J., Two-fluid and population balance models for subcooled boiling flow. Applied Mathematical Modelling, 30, pp. 1370-1391, 2006.

[11] Bo, T., CFD homogeneous mixing flow modelling to simulate subcooled nucleate boiling flow. SAE International, 2004-01-1512, 2004.

[12] Przulj, V., Birkby, P. \& Mason, P., Finite volume method for conjugate heat transfer in complex geometries using cartesian cut-cell grids. CHT-08, Marrakech, Morocco, 2008.

[13] Robinson, K., IC Engine Coolant Heat Transfer Studies. PhD thesis, University of Bath, Department of Mechanical Engineering, 2001. 